\title{
MANIFOLDS WITH FEW CELLS AND THE STABLE HOMOTOPY OF SPHERES
}

\author{
LARRY SMITH
}

\begin{abstract}
Let $f: S^{n+k-1} \rightarrow S^{n}$ and form the complex $V(f)=$ $S^{n} \vee S^{k} \cup_{f+\left[i_{n}, i_{k}\right]} e^{n+k}$ where $i_{t} \in \pi_{t}\left(S^{t}\right)$ is the canonical generator and [ ] denotes Whitehead product. The complex $V(f)$ is a Poincare duality complex. Under the assumption that $f$ is in the stable range we show that $V(f)$ has the homotopy type of a smooth, combinatorial or topological manifold iff the map $f$ lies in the image of the $O$, PL or Top $J$-homomorphism respectively.
\end{abstract}

Let $\pi_{*}^{S}$ denote the stable homotopy groups of spheres and suppose that $\varphi \in \pi_{k-1}^{S}$. We may represent $\varphi$ by a map $f: S^{n+k-1} \rightarrow S^{n}$, and form the complex $X(f)=S^{n} \cup_{f} e^{n+k}$. By studying the complex $X(f)$ we may often detect that $\varphi \neq 0$. For example in this way one may study the Hopf invariant, $e_{\mathrm{C}}$-invariant, etc. [1], [2], [9]. We may also form the complex

$$
V(f)=S^{n} \vee S^{k} \cup_{f+\left[i_{n}, i_{k}\right]} e^{n+k}
$$

where $i_{t} \in \pi_{t}\left(S^{t}\right)$ is the canonical generator. The complex $V(f)$ is in fact a Poincaré duality complex, and it is therefore to be hoped that by asking questions about smoothing $V(f)$ we may discover information about the element $\varphi$ and vice versa. The present note is devoted to a small stab in this direction.

Our main result is the following:

THEOREM. Suppose that $\varphi \in \pi_{k-1}^{S}$. Represent $\varphi$ by a map $f: S^{n+k-1} \rightarrow S^{n}$, $n \gg k$, and form the complex

$$
V(f)=S^{n} \vee S^{k} \cup_{f+\left[i_{n}, i_{k}\right]} e^{n+k} .
$$

Then $V(f)$ has the homotopy type of a closed topological, combinatorial or smooth manifold, iff $\varphi$ lies in the image of the homomorphism $J_{\mathrm{T} o \mathrm{p}}, J_{\mathrm{PL}}$ or $J_{O}$ respectively.

We make no claim for originality for the preceding theorem. The result should be well known on the basis of the results of [5], but it does

Received by the editors November 6, 1970 .

AMS 1969 subject classifications. Primary 5540, 5560.

Key words and phrases. Smoothing Poincaré complexes, the $J$-homomorphism.

(C) American Mathematical Society 1972 
not seem to be. Perhaps there is some interest in rephrasing the results of [5] so as to apply to modern smoothing problems.

If $V(f)$ actually admits a homotopy smoothing, then it bounds as a smooth manifold. However, if $V(f)$ admits a homotopy almost complex structure then it is possible to calculate $e_{\mathbf{C}}(\varphi) \in \mathbf{Q} / Z$ by studying the natural map $V(f) \rightarrow X(f)$ and its Todd character (see for example [7]). It would be of interest to define an invariant of Poincaré complexes which applied to $V(f)$ yielded $e_{\mathrm{C}}(\varphi)$ or the Hopf invariant of $\varphi$.

We turn now to the proof of the preceding theorem. Let us denote by $B F$ the classifying space for spherical fibrations. Recall that there is a natural isomorphism $\pi_{*}^{S} \cong \pi_{*+1}(B F)$. Thus we may represent $\varphi$ by a map $g: S^{k} \rightarrow B F$. This map determines, with $n \gg k$, a spherical fibration

with fibre $S^{n}$.

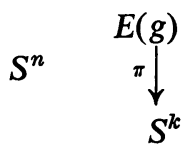

Proposition 1. With the preceding notations, $E(g)$ and $V(f)$ have the same homotopy type.

Proof. First we consider the exact homotopy sequence of $\pi$. We have

$$
\rightarrow \pi_{k}(E(g)) \stackrel{\pi_{*}}{\rightarrow} \pi_{k}\left(S^{k}\right) \rightarrow \pi_{k-1}\left(S^{n}\right)=0
$$

since $n \gg k$. Hence there exists $\sigma \in \pi_{k}(E(g))$ such that $\pi_{*}(\sigma)=1 \in \pi_{k}\left(S^{k}\right)$. By the covering homotopy theorem we may find a map $s: S^{k} \rightarrow E(g)$ that represents $\sigma \in \pi_{k}(E(g))$ and is a cross-section to $\pi$. Thus we receive a map $S^{n} \vee S^{k} \hookrightarrow E(g)$ from $s$ and the inclusion of a fibre $S^{k} \hookrightarrow E(g)$. Therefore $E(g)$ has a cell structure

$$
E(g)=S^{n} \vee S^{k} \cup_{\mu} e^{n+k} .
$$

By the Hilton-Milnor theorem [3],

$$
\pi_{n+k-1}\left(S^{n} \vee S^{k}\right)=\mathbf{Z} \oplus \pi_{n+k-1}\left(S^{n}\right) \oplus \pi_{n+k-1}\left(S^{k}\right)
$$

where the infinite cyclic summand is generated by the Whitehead product $\left[i_{n}, i_{k}\right]$. Therefore

$$
\mu=m\left[i_{n}, i_{k}\right]+\alpha+\beta
$$

where $m \in Z, \alpha \in \pi_{n+k-1}\left(S^{n}\right), \beta \in \pi_{n+k-1}\left(S^{k}\right)$. The Gysin sequence for the fibration $\pi: E(g) \rightarrow S^{k}$ shows that the cup product induces an isomorphism

$$
\cup: H^{k}(E(g) ; Z) \otimes H^{n}(E(g) ; Z) \rightarrow H^{n+k}(E(g) ; Z) .
$$

Therefore, by a theorem of James $[4,(6.2),(6.3)], m= \pm 1$. Since 
$s: S^{n} \hookrightarrow E(g)$ is a retract $\beta=0$ and so we may assume

$$
\mu=\left[i_{n}, i_{k}\right]+\alpha: \alpha \in \pi_{n+k-1}\left(S^{n}\right),
$$

and it remains to compute $\alpha$. To this end let $\omega: S^{n} \times S^{k-1} \rightarrow S^{n}$ be the adjoint of the map

$$
\partial g: S^{k-1} \rightarrow F(n)=\operatorname{Map}\left(S^{n}, S^{n}\right)
$$

where $\partial: \pi_{*}(B F(n)) \rightarrow \pi_{*-1}(F(n))$ is the natural isomorphism. Let $H(\omega): S^{n+k} \rightarrow S^{n+1}$ be the map obtained by applying the Hopf construction to $\omega$. It is known that under the usual isomorphism of $\pi_{*}^{S}$ with $\pi_{*}(F)$ that $H(\omega)=\varphi \in \pi_{k-1}^{S}$ (recall we are in the stable range since $n \gg k$ ). The work of James and Whitehead [5, 3.9] as modified for spherical fibrations shows $\alpha= \pm H(\omega)$. Since we are in the stable range this means that $f: S^{n+k} \rightarrow S^{n}$ may be chosen to represent $\alpha$ and therefore

$$
\mu=\left[i_{n}, i_{k}\right]+[f]
$$

which yields the desired conclusion.

Proposition 2. With the preceding notations $V(f)$ is a Poincaré duality complex.

First Proof. This follows from Proposition 1 and the Gysin sequence of the spherical fibration $E(g) \stackrel{\pi}{\rightarrow} S^{k}$.

SECOND Proof. This is a special case of the theorem of James [4] already cited.

The following result is an analog of a result of Spivak [8, 7.6, 8.3].

Proposition 3. With the preceding notations, the first obstruction to lifting the Spivak normal map [8] $v: V(f) \rightarrow B F$ to BTop, BPL or $B O$ is the coset of $\varphi$ in $\pi_{*}^{S} / \operatorname{Im} J_{\mathrm{Top}}, \pi_{*}^{S} / \operatorname{Im} J_{\mathrm{PL}}$ or $\pi_{*}^{S} / \operatorname{Im} J_{O}$ respectively.

Proof. As the proofs in the three cases are similar we present the details for the smooth case only. We are concerned with the lifting problem

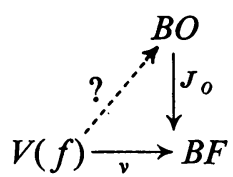

By standard obstruction theory the first obstruction to constructing the indicated lifting lies in

$$
H^{k}\left(V(f) ; \pi_{k-1}(F / O)\right) \cong \pi_{k-1}(F / O) .
$$

Denote this obstruction by $\mathcal{O}(f)$. Let

$$
\pi_{k}(B O) \underset{J_{o}}{\longrightarrow} \|_{\pi_{k-1}^{S}} \pi_{k}(B F) \underset{\Delta}{\longrightarrow} \pi_{k-1}(F / O)
$$


be a portion of the homotopy exact sequence of the fibring

Let $^{1}$

$$
\mathrm{F} / \mathrm{O} \rightarrow \mathrm{BO} \stackrel{\mathrm{JO}^{\circ}}{\longrightarrow} \mathrm{BF} .
$$

$$
\psi: H^{n}(V(f) ; Z) \rightarrow H^{n+k}\left(V(f) ; \pi_{n+k-1}\left(S^{k}\right)\right)
$$

be the cohomology operation defined by Kervaire and Milnor in [6]. If $e_{n} \in H^{n}(V(f) ; Z)$ is a generator then clearly $\psi e_{n}=[f] \in \pi_{k-1}^{S}$ where we have identified

$$
H^{n+k}\left(V(f) ; \pi_{n+k-1}\left(S^{k}\right)\right) \cong \pi_{n+k-1}\left(S^{k}\right) \cong \pi_{k-1}^{S}
$$

by the universal coefficient theorem and Freudenthal suspension theorem. Let $\psi^{k}(f) \in H^{k}\left(V(f) ; \pi_{k-1}^{S}\right)$ be the unique class such that

$$
\psi(c)=c \cup \psi^{k}(f): c \in H^{n}(V(f) ; Z) .
$$

Then our above computation shows that $\psi^{k}(f)=[f]=\varphi \in \pi_{k-1}^{S}$ where we have again identified $H^{k}\left(V(f) ; \pi_{k-1}^{S}\right) \cong \pi_{k-1}^{S}$. Let

$$
\Delta_{*}: H^{k}\left(V(f) ; \pi_{k-1}^{S}\right) \rightarrow H^{k}\left(V(f) ; \pi_{k-1}(F / O)\right)
$$

be the map induced by the coefficient homomorphism

$$
\Delta: \pi_{k}(B F)=\pi_{k-1}^{S} \rightarrow \pi_{k-1}(F / O) .
$$

According to Spivak [8, 8.3], $\Delta_{*} \psi^{k}(f)=\mathcal{O}(f)$. Identifying

$$
H^{k}\left(V(f) ; \pi_{k-1}(F / O)\right)=\pi_{k-1}(F / O)
$$

and reflecting on the exactness of the sequence

$$
\pi_{k}(B O) \underset{J_{o}}{\longrightarrow} \pi_{k-1}^{S} \underset{\Delta}{\longrightarrow} \pi_{k-1}(F / O)
$$

yields the desired conclusion.

Proof of Theorem. As the proof is similar in the topological, combinatorial, or smooth case we give the details for the smooth case only.

Suppose that $\varphi \in \operatorname{Im}\left\{J_{O}: \pi_{*}(B O) \rightarrow \pi_{*}^{S}\right\}$. Then we may assume that $g: S^{k} \rightarrow B F$ factors through $B O$ as follows

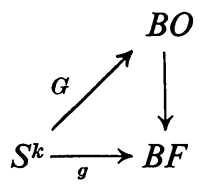

${ }^{1}$ Suppose that $X$ is a finite complex satisfying $H^{i}(X ; G)=0: r<i<s$ for all coefficient groups $G$, where $2<r<s-2$. The operation [8] $\psi: H^{r}(X ; Z) \rightarrow$ $H^{s}\left(X ; \pi_{r+8-1}\left(S^{r}\right)\right)$ is defined by $\psi(x)=$ the first obstruction to the existence of map $\sigma: X \rightarrow S^{r}$ such that $\sigma^{*}(i)=x$, where $i \in H^{r}\left(S^{r} ; Z\right)$ is the canonical generator. 
The spherical fibre space $\pi: E(g) \rightarrow S^{k}$ has therefore the fibre homotopy type of the sphere bundle with stable classifying map $G: S^{k} \rightarrow B O$. Since $S^{k}$ is compact we may replace $B O$ by a smooth closed manifold. The map $G$ may then be smoothed in its homotopy class so that $E(g)$ has the homotopy type of the total space of a smooth sphere bundle over $S^{k}$ and hence $E(g)$ is of the homotopy type of a smooth manifold.

Suppose next that $V(f)$ admits a smoothing. Let $V(f) \stackrel{v}{\rightarrow} B F$ represent the Spivak normal fibre space of $V(f)$. From smoothing theory it follows [10] that there is a factorization

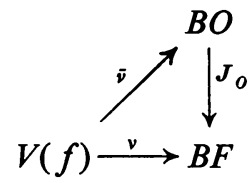

and the result follows directly from Proposition 3.

It is perhaps of interest to note that the manifolds $V(f)$ admit maps of degree $d$ for arbitrary integers $d$. This answers in the negative a question raised by M. Agoston at the 1970 AMS Summer Institute in Algebraic Topology.

To construct a map $\varphi_{d}: V(f) \rightarrow V(f)$ of degree $d \in \mathbf{Z}$ consider the following diagram

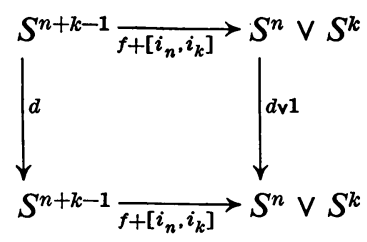

Since we are in the stable range we have

$$
d\left(f+\left[i_{n}, i_{k}\right]\right)=d f+d\left[i_{n}, i_{k}\right]
$$

and by the bilinearity of Whitehead products

$$
\left(f+\left[i_{n}, i_{k}\right]\right)(d \vee 1)=d f+\left[d i_{n}, i_{k}\right]=d f+d\left[i_{n}, i_{k}\right] .
$$

Therefore the preceding square commutes and so we obtain a diagram wherein the rows are cofibrations

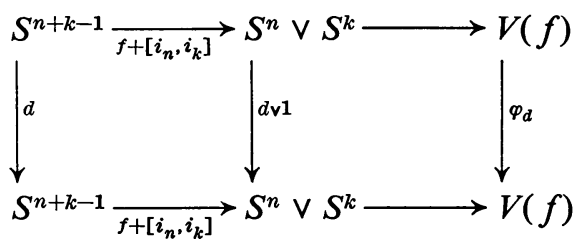


It is routine to check that $\varphi_{d}$ has degree $d$. If then [ $\left.f\right]=\varphi$ lies in the image of $J_{O}, J_{\mathrm{PL}}$, or $J_{\text {Top }}$ we obtain a smooth, combinatorial or topological manifold admitting maps of arbitrary degree.

\section{REFERENCES}

1. J. F. Adams, On the non-existence of elements of Hopf invariant one, Ann. of Math. (2) 72 (1960), 20-104. MR 25 \#4530.

2. - On the groups $J(X)$. IV, Topology 5 (1966), 21-71. MR 33 \#6628.

3. P. J. Hilton, On the homotopy groups of the union of spheres, J. London Math. Soc. 30 (1955), 154-172. MR 16, 847.

4. I. M. James, Note on cup-products, Proc. Amer. Math. Soc. 8 (1957), 374-383. MR 19, 974.

5. I. M. James and J. H. C. Whitehead, The homotopy theory of sphere bundles over spheres. I, Proc. London Math. Soc. (3) 4 (1954), 196-218. MR 15, 892.

6. M. Kervaire and J. W. Milnor, Groups of homotopy spheres. I, Ann. of Math. (2) 77 (1963), 504-537. MR 26 \#5584.

7. L. Smith, The Todd character, the Chern character and elementary integrality theorems, Illinois J. Math. (to appear).

8. M. Spivak, Spaces satisfying Poincaré duality, Topology 6 (1967), 77-101. MR 35 \#4923.

9. N. E. Steenrod, Cohomology invariants of mappings, Ann. of Math. (2) 50 (1949), 954-988. MR 11, 122.

10. C. T. C. Wall, Surgery on compact manifolds, Academic Press, New York, 1971.

Department of Mathematics, University of Virginia, Charlottesville, VirGINIA 22903 